\title{
Erratum: Geometric effects resulting from square and circular confinements for a particle constrained to a space curve [Phys. Rev. A 97, 042108 (2018)]
}

\author{
Yong-Long Wang, Meng-Yun Lai, Fan Wang, Hong-Shi Zong, and Yan-Feng Chen
}

(Received 5 June 2018; published 20 June 2018)

DOI: 10.1103/PhysRevA.97.069904

In our recently published paper there were typographical errors appearing in Eqs. (5), (6), (16), (23), (30), and (32). That is $f^{-(1 / 2)}$ was mistakenly typed as $f^{1 / 2}$, and $f^{1 / 2}$ was mistakenly typed as $f^{-(1 / 2)}$. Under rescaled transformation, an introduced new wave function $\chi$ and a physical operator $\hat{\mathrm{F}}$, using the rescaled factor $f$, can be expressed as [1]

$$
\chi=f^{1 / 2} \psi,
$$

and

$$
\hat{\mathrm{F}}^{\prime}=f^{1 / 2} \hat{\mathrm{F}} f^{-(1 / 2)} .
$$

Equation (5) and its related expression are to be corrected. In the spirit of the confining potential formalism, for a particle confined to a space curve, the effective form of $\hat{F}$ can be determined by

$$
\hat{\mathrm{F}}_{\text {eff }}=\lim _{q_{N} \rightarrow 0}\left(\hat{\mathrm{F}} f^{-(1 / 2)}\right)-\hat{\mathrm{F}}_{N}=\lim _{q_{N} \rightarrow 0}\left(1 \hat{\mathrm{F}} f^{-(1 / 2)}\right)-\hat{\mathrm{F}}_{N}=\lim _{q_{N} \rightarrow 0}\left(f^{1 / 2} \hat{\mathrm{F}} f^{-(1 / 2)}\right)-\hat{\mathrm{F}}_{N} .
$$

Following the first equality, $f^{-(1 / 2)}$ is from the relationship $\psi=f^{-(1 / 2)} \chi$, which is determined by the normalization condition $\int d s d q_{2} d q_{3} \sqrt{g}\left|f^{1 / 2} \psi\right|^{2}=\int d s d q_{2} d q_{3} \sqrt{g}|\chi|^{2}=1$. Here $\lim _{q_{N} \rightarrow 0} f^{1 / 2}=1$ is considered. Equation (6) should read

$$
\hat{\mathrm{F}}_{\text {eff }}=\lim _{\varepsilon_{c} \rightarrow 0}\left\langle\chi_{0_{c}}\left|f^{1 / 2} \hat{\mathrm{F}} f^{-(1 / 2)}-\hat{\mathrm{F}}_{N}\right| \chi_{0_{c}}\right\rangle=\left\langle\chi_{0_{c}}\left|f^{1 / 2} \hat{\mathrm{F}} f^{-(1 / 2)}-\hat{\mathrm{F}}_{N}\right| \chi_{0_{c}}\right\rangle_{0},
$$

Eq. (16) should be

$$
\begin{aligned}
\hat{\mathrm{H}}_{\mathrm{eff}} & =\left\langle\chi_{0_{n}, 0_{b}}\left|f^{1 / 2} \hat{\mathrm{H}}_{V} f^{-(1 / 2)}-\hat{\mathrm{H}}_{N}\right| \chi_{0_{n}, 0_{b}}\right\rangle_{0} \\
& =\lim _{w \rightarrow \infty}\left\langle\chi_{0_{n}, 0_{b}}\left|f^{1 / 2} \hat{\mathrm{H}}_{V} f^{-(1 / 2)}-\hat{\mathrm{H}}_{N}\right| \chi_{0_{n}, 0_{b}}\right\rangle \\
& =\frac{1}{2 m} \hat{p}_{s}^{2}-\frac{\hbar^{2}}{8 m} \kappa^{2}-\frac{\hbar^{2}}{4 m} \tau^{2},
\end{aligned}
$$

Eq. (23) as

$$
\hat{\mathrm{H}}_{\mathrm{eff}}=\left\langle\chi_{0, l}\left|f^{1 / 2} \hat{H}_{V} f^{-(1 / 2)}-\hat{\mathrm{H}}_{N}\right| \chi_{0, l}\right\rangle_{0}=\frac{1}{2 m}\left(\hat{p}_{s}-l \hbar \tau\right)^{2}-\frac{\hbar^{2}}{8 m} \kappa^{2},
$$

Eq. (30) as

$$
\overrightarrow{\mathrm{p}}_{\text {eff }}=\left\langle\chi_{0_{n}, 0_{b}}\left|f^{1 / 2} \overrightarrow{\mathrm{p}} f^{-(1 / 2)}-\overrightarrow{\mathrm{p}}_{N}\right| \chi_{0_{n}, 0_{b}}\right\rangle_{0}=\overrightarrow{\mathrm{p}}_{s}+\overrightarrow{\mathrm{p}}_{g}=-i \hbar\left(\vec{t} \partial_{s}-\frac{1}{2} \kappa \vec{n}\right),
$$

and Eq. (32) as

$$
\overrightarrow{\mathrm{p}}_{\text {eff }}=\left\langle\chi_{0, l}\left|f^{1 / 2} \overrightarrow{\mathrm{p}} f^{-(1 / 2)}-\overrightarrow{\mathrm{p}}_{N}\right| \chi_{0, l}\right\rangle_{0}=\overrightarrow{\mathrm{p}}_{s}
$$

This work was supported, in part, by the National Major State Basic Research and Development of China (Grant No. 2016YFE0129300), the National Key R and D Program of China (Grant No. 2017YFA0303700), the National Nature Science Foundation of China (Grants No. 11625418, No. 11474158, No. 51472114, No. 11404157, No. 11475085, and No. 11535005). Y.-L.W. was funded by Linyi University (Grant No. LYDX2016BS135), the Natural Science Foundation of Shandong Province of China (Grant No. ZR2017MA010), and the China Postdoctoral Science Foundation (Grant No. 2017M611770). 